Supporting Information

\title{
Bifunctional Gold Nanobipyramids for Photothermal Therapy and Temperature Monitoring
}

\author{
Xueke Wu, ${ }^{\text {a,b }}$ Lixuan Mu, ${ }^{*, a}$ Min Chen, ${ }^{\text {a,b }}$ Sen Liang, ${ }^{\text {a,b }}$ Yuan Wang, ${ }^{\text {a,b }}$ Guangwei \\ She, ${ }^{\mathrm{a}}$ and Wensheng Shi*,a,b \\ ${ }^{a}$ Key Laboratory of Photochemical Conversion and Optoelectronic Materials, \\ Technical Institute of Physics and Chemistry, Chinese Academy of Sciences, Beijing \\ 100190, China \\ E-mail:mulixuan@mail.ipc.ac.cn; shiws@mail.ipc.ac.cn; \\ Fax:+86-10-82543513; Tel: +86-10-82543513 \\ ${ }^{b}$ University of Chinese Academy of Sciences, Beijing 100049, China
}


In the second step of the AuNBPs synthesis, it was found that the color of the solution changed from colorless to purple gradually after the seed solution was added into the mixture solution. It is known that the color of the solution strongly depended on the morphology of the nanoparticle. Accordingly, the morphology of the as-grown gold nanostructures in this step was investigated. The AuNBPs and irregular AuNPs can be observed from the as-grown gold nanostructures (Figure S1a). In order to separate the AuNBPs and irregular AuNPs, the as-grown gold nanostructures were incubated with $\mathrm{AgNO}_{3}$ at $65{ }^{\circ} \mathrm{C}$ and the core-shell structures of the $\mathrm{Au} / \mathrm{AgNRs}$ and $\mathrm{Au} / \mathrm{AgNPs}$ could be developed from the AuNBPs and irregular AuNPs respectively in the solution. ${ }^{1}$ Moreover, the Au/AgNRs tend to be precipitated to the bottom. While, the $\mathrm{Au} / \mathrm{AgNPs}$ could still suspended in the solution. ${ }^{1}$ Removing the supernatant, only the Au/AgNRs were left. Their morphologies were confirmed by TEM, and shown in Figure S1b and S1c, respectively. Furthermore, the structure of the Au/AgNRs was confirmed by the maps of the elemental distribution. The silver and gold element distribution within the $\mathrm{Au} / \mathrm{AgNRs}$ were indicated by the colors of the green and purple, respectively, in Figure S2, revealing that the $\mathrm{Au} / \mathrm{AgNRs}$ own the structure of the $\mathrm{Au}$ core and Ag shell. After the Ag was etched from the the Au/AgNRs and Au/AgNPs, the pure AuNBPs and irregular AuNPs can be obtained, and their morphologies were shown in Figure S3a, S3b.
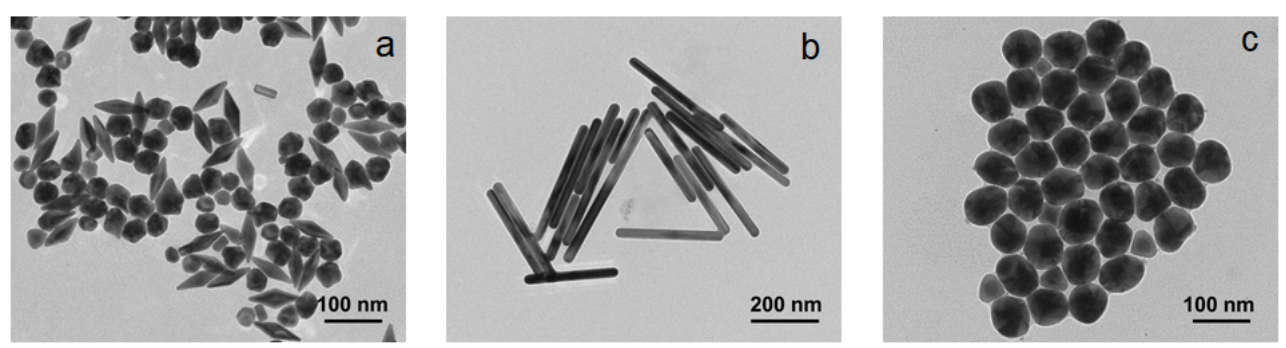

Figure S1. TEM images of (a) as-grown gold nanostructures, (b) Au/AgNRs, (c) Au/AgNPs. 

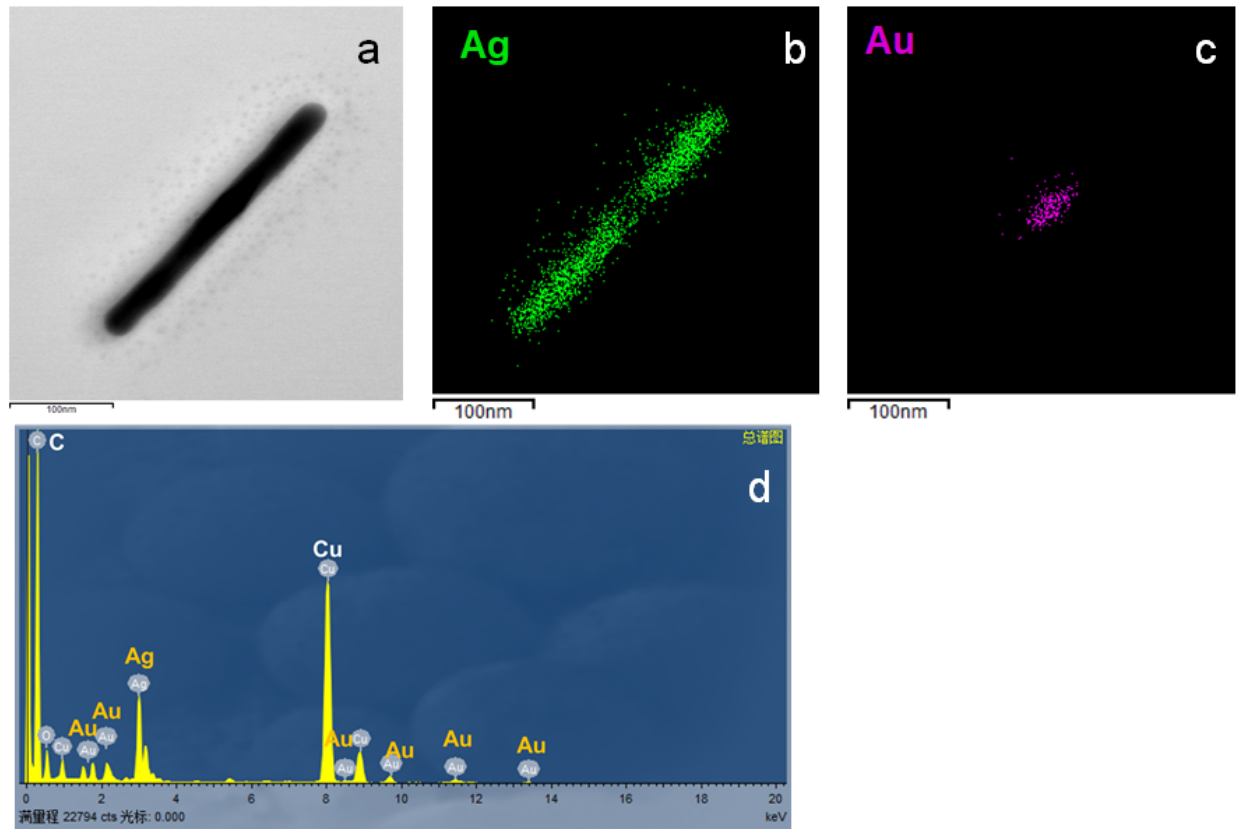

Figure S2. (a) The TEM image of the Au/AgNRs, (b) (c) elemental mapping of the core/shell Au/AgNRs, (d) The elemental analysis of the Au/AgNRs.
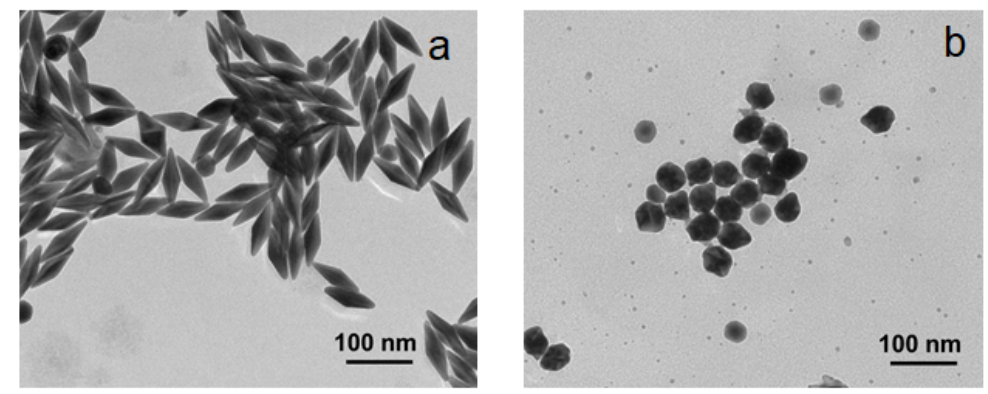

Figure S3. TEM images of (a) the pure AuNBPs, (b) the irregular AuNPs.

The absorption spectra of the five kinds of particles as mentioned above were shown in Figure S4. The as-grown gold nanostructures were exhibited with two absorption peaks around $550 \mathrm{~nm}$ and $800 \mathrm{~nm}$, which correspond with the irregular AuNPs and AuNBPs in the sample. ${ }^{1}$ The absorption peak at $550 \mathrm{~nm}$ obviously disappeared in the absorption of the AuNBPs comparing with the spectrum of as-grown gold nanostructures. The absorption of the irregular AuNPs only have the peak of 550 $\mathrm{nm}$. Accordingly, the disappearance of the peak at $550 \mathrm{~nm}$ in the absorption of the AuNBPs can further verify that few irregular particles were in the solution. This result can be further confirmed by the corresponding TEM images (Figure S1a, S2a). 


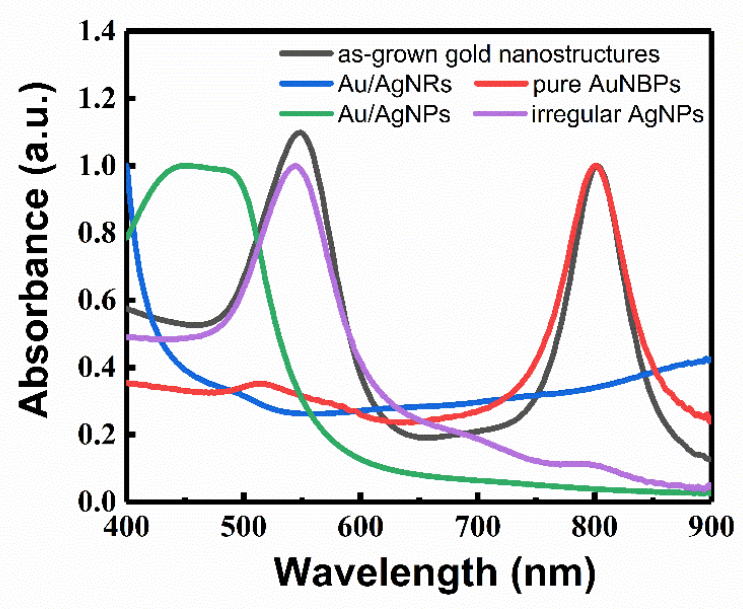

Figure S4. The absorption spectra of the five kinds of the particles

The photographs of these five kinds of the particles were shown in Figure. S5. Obviously, the color of the particles with different morphologies are distinctly diverse. Furthermore, the color can be employed to deduce the particle morphology.

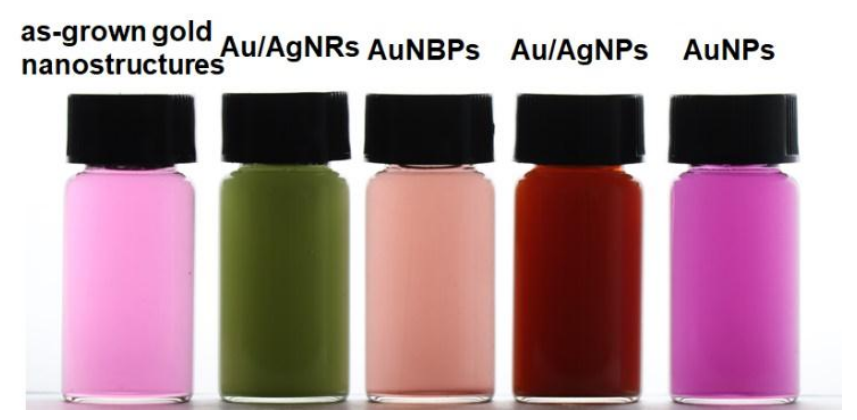

Figure S5. The photographs of the five kinds of the particles

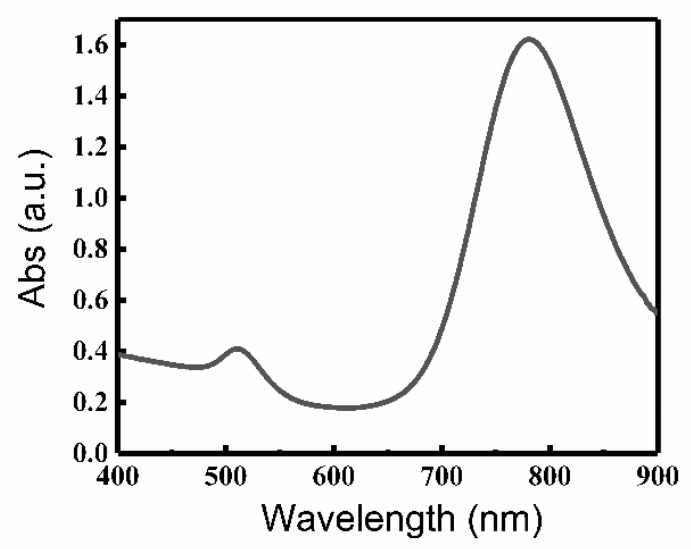

Figure S6. The absorption spectrum of the selected AuNRs. 

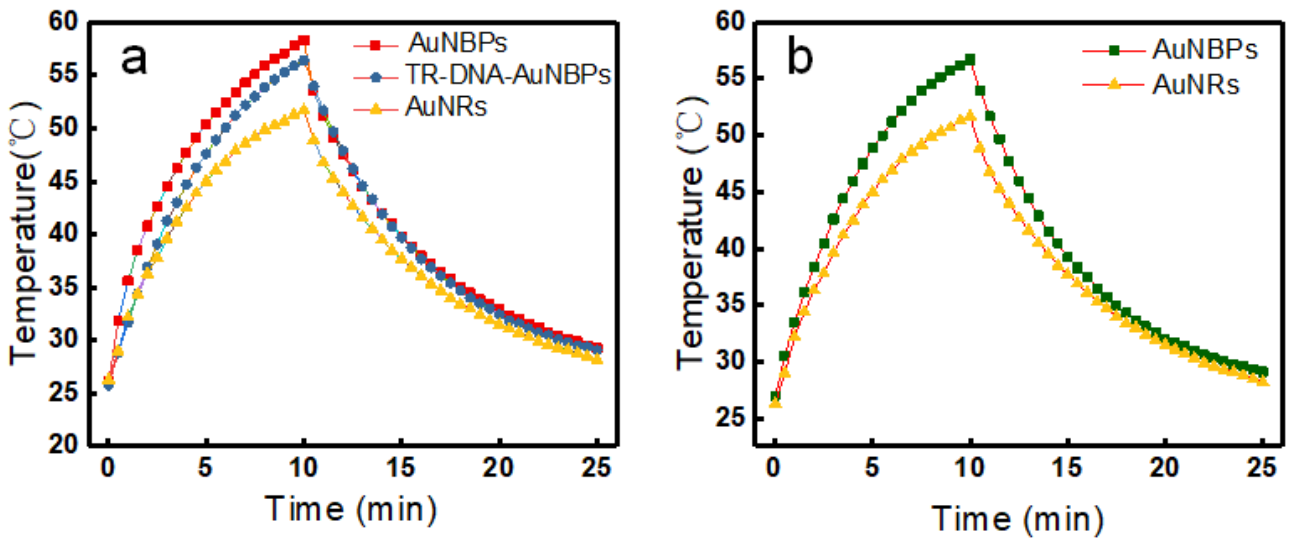

Figure S7. (a) The temperature variation of the AuNBPs, AuNRs and TR-DNA-AuNBPs solution $\left(30 \mu \mathrm{g} \cdot \mathrm{mL}^{-1}\right)$ under the irradiation with an $808 \mathrm{~nm}$ laser at $1.0 \mathrm{~W} \cdot \mathrm{cm}^{2}$ for $10 \mathrm{~min}$, and cooled naturally for 15min. (b) The course of the temperature of the AuNBPs and AuNRs with the same absorbance of 1.82 under the irradiation with an $808 \mathrm{~nm}$ laser at $1.0 \mathrm{~W} \cdot \mathrm{cm}^{2}$ for $10 \mathrm{~min}$, and cooled naturally for $15 \mathrm{~min}$.

Table S1 Comparison of different photothermal materials

\begin{tabular}{|c|c|c|c|c|}
\hline Photothermal materials & $\begin{array}{c}\text { Photothermal } \\
\text { conversion efficiency(n) }\end{array}$ & $\begin{array}{c}\text { LPRW } \\
(\mathbf{n m})\end{array}$ & $\begin{array}{c}\text { Laser } \\
(\mathbf{n m})\end{array}$ & Reference \\
\hline Gold nanocages & $30.1 \%$ & 808 & 808 & S2 \\
\hline Gold nanoshells & $30.2 \%$ & 808 & 808 & S2 \\
\hline $\begin{array}{c}\text { Gold nanostars Coated Hollow } \\
\text { Mesoporous Silica }\end{array}$ & $67.1 \%$ & 795 & 808 & S3 \\
\hline SnS & $39.4 \%$ & 705 & 785 & S4 \\
\hline Gold nanostars & $48.43 \%$ & 808 & 808 & S5 \\
\hline Gold nanorods & 250\% & $798-807$ & 808 & S6 \\
\hline Gold nanorods & $62 \%$ & 800 & 808 & This work \\
\hline
\end{tabular}




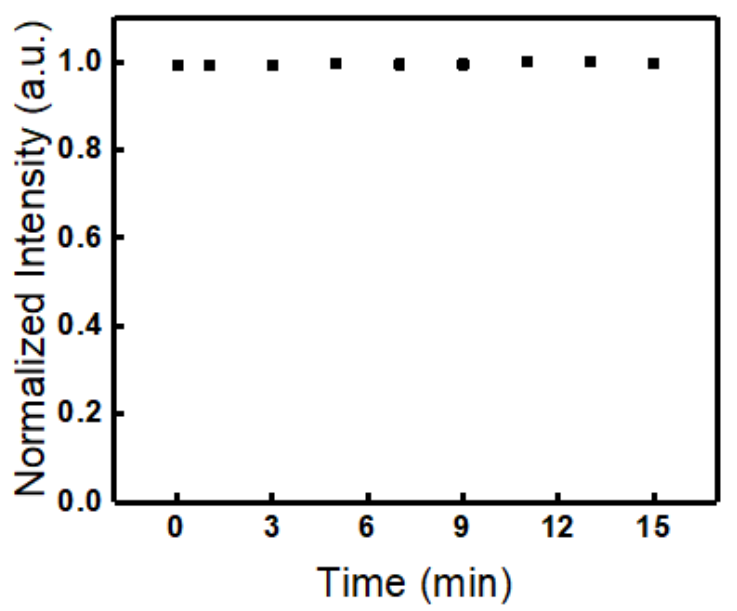

Figure S8. The normalized FI of the DNA-TR under different irradiation time. (laser of $808 \mathrm{~nm}, 1$ $\left.\mathrm{W} \cdot \mathrm{cm}^{-1}\right)$

\section{REFERENCES}

1. Li, Q.; Zhuo, X.; Li, S.; Ruan, Q.; Xu, Q.-H.; Wang, J., Production of Monodisperse Gold Nanobipyramids with Number Percentages Approaching 100\% and Evaluation of Their Plasmonic Properties. Advanced Optical Materials 2015, 3 (6), 801-812.

2. Feng, Y.; Chang, Y.; Sun, X.; Cheng, Y.; Zheng, R.; Wu, X.; Wang, L.; Ma, X.; Li, X.; Zhang, H., Differential photothermal and photodynamic performance behaviors of gold nanorods, nanoshells and nanocages under identical energy conditions. Biomaterials Science 2019, 7 (4), 1448-1462.

3. Li, X.; Xing, L.; Zheng, K.; Wei, P.; Du, L.; Shen, M.; Shi, X., Formation of Gold Nanostar-Coated Hollow Mesoporous Silica for Tumor Multimodality Imaging and Photothermal Therapy. Acs Applied Materials \& Interfaces 2017, 9 (7), 5817-5827.

4. Chen, F.; Yang, D.; Shen, H.; Deng, M.; Zhang, Y.; Zhong, G.; Hu, Y.; Weng, L.; Luo, Z.; Wang, L., Hydrothermal synthesis of novel rhombic dodecahedral $\mathrm{SnS}$ nanocrystals for highly efficient photothermal therapy. Chemical Communications 2019, 55 (19), 2789-2792.

5. Wu, D.; Fan, M.; Zhang, L.; Xing, J.; Lyu, S.; Zeng, L., Controllable Synthesis,Photothermal Conversion and in vitro Photothermal Therapy of Gold Nanostars /Nanobipyramids. Chinese Journal of Luminescence 2018, 39 (3), 280-286.

6. Almada, M.; Leal-Martinez, B. H.; Hassan, N.; Kogan, M. J.; Burboa, M. G.; Topete, A.; Valdez, M. A.; Juarez, J., Photothermal conversion efficiency and cytotoxic effect of gold nanorods stabilized with chitosan, alginate and poly(vinyl alcohol). Materials Science \& Engineering C-Materials for Biological Applications 2017, 77, 583-593. 